\title{
Câncer infantil e impactos emocionais para a família: Uma revisão da literatura
}

\author{
Childhood cancer and emotional impacts on the family: A review of the literature \\ Cáncer infantil e impactos emocionales en la familia: Una revisión de la literatura
}

Recebido: 06/07/2021 | Revisado: 13/07/2021 | Aceito: 29/07/2021 | Publicado: 05/08/2021

\author{
Jaimeson Araújo de Souza \\ ORCID: https://orcid.org/0000-0001-9910-5424 \\ Centro Universitário de Patos, Brasil \\ E-mail: jaimesonsouza@hotmail.com \\ José Ytalo de Figueiredo Alves Campos \\ ORCID: https://orcid.org/0000-0001-5378-3502 \\ Centro Universitário de Patos, Brasil \\ E-mail: ytalofac@gmail.com \\ Francisco Theotonio dos Santos Neto \\ ORCID: https://orcid.org/0000-0002-5820-3957 \\ Centro Universitário de Patos, Brasil \\ E-mail: fnetotheotonio@gmail.com \\ Mayara Nóbrega Araújo \\ ORCID: https://orcid.org/0000-0003-4126-1402 \\ Centro Universitário de Patos, Brasil \\ E-mail: mayara38araujo38@gmail.com \\ Milena Nunes Alves de Sousa \\ ORCID: https://orcid.org/0000-0001-8327-9147 \\ Centro Universitário de Patos, Brasil \\ E-mail: milenanunes@ fiponline.edu.br
}

\begin{abstract}
Resumo
O câncer infantojuvenil, definido como câncer em crianças e adolescentes com idade entre 0-19 anos, gera impactos profundos na vida de todos, sendo um grande indutor de sentimentos negativos na criança, adolescente e nas pessoas próximas. Assim, propôs-se analisar os impactos emocionais do câncer infantil para a família, discutindo os diversos sentimentos despertados no núcleo familiar pelo processo de tratamento e eventual cura do câncer infantojuvenil. O presente estudo está estruturado por meio de uma revisão integrativa da literatura, através da elaboração da seguinte pergunta norteadora: "Quais os impactos emocionais do câncer infantil para a família?"; a definição de bancos de dados como PUBMED, SCIELO e BVS que serviram como instrumento de pesquisa; tomando por base os descritores, em inglês: neoplasm, child, family e emotions, por não apresentar resultados nas bases de pesquisa com os descritores em português; E dos critérios de elegibilidade e ainda uma adequação aos critérios de inclusão e exclusão definidos. Com relação aos resultados, as famílias relataram inúmeros impactos na qualidade de vida do núcleo familiar, distúrbios psicológicos, dificuldades nas relações entre os pais e a mudança na dinâmica familiar em virtude do tratamento da criança com câncer pediátrico. Portanto, entre as emoções negativas destacaram-se o medo, irritabilidade, ansiedade, incerteza e tristeza; quanto às positivas, a resiliência, suporte social, fé, esperança, abertura, amor e positividade. Dessa forma, o diagnóstico e posterior tratamento do câncer infantil é um momento muito delicado para toda a família e o acompanhamento psicológico para a criança e familiares é essencial para minimizar os efeitos deletérios causados pelos sentimentos ruins e ampliar o alcance dos bons sentimentos.
\end{abstract}

Palavras-chave: Neoplasias; Diagnóstico; Comportamento infantil; Cuidadores; Emoções manifestas.

\begin{abstract}
Childhood cancer, defined as cancer in children and adolescents aged 0-19 years, has profound impacts on everyone's lives, being a great inducer of negative feelings in children, adolescents and those close to them. So, the aim of the present review is to analyze the emotional impacts of childhood cancer on the family, discussing the different feelings aroused in the family nucleus by the treatment process and eventual cure of childhood cancer. This study is structured by an integrative literature review, based on the elaboration of the following guiding question: "What are the emotional impacts of childhood cancer on the family?"; the definition of databases such as PUBMED, SCIELO and VHL that served as a research instrument; based on the descriptors: Neoplasm, Child, Family, Emotions and the eligibility criteria for the review. Regarding the results, families reported numerous impacts on the Family such as psychological disorders, difficulties in relationships between parents and the change in family dynamics due to the treatment of children with pediatric cancer. Therefore, among the negative emotions, fear, irritability, anxiety, uncertainty and sadness stood out; as for the positive ones, resilience, social support, faith, hope, openness, love and positivity. The diagnosis and subsequent treatment of childhood cancer is a very delicate moment for the whole
\end{abstract}


family and psychological support for the child and the family is essential to minimize the harmful effects caused by bad feelings and expand the reach of good feelings.

Keywords: Neoplasm; Diagnostic; Childish behavior; Caregivers; Manifest emotions.

\begin{abstract}
Resumen
El cáncer infantil, definido como cáncer en niños y adolescentes de 0 a 19 años, tiene profundos impactos en la vida de todas las personas, siendo un gran inductor de sentimientos negativos en niños, adolescentes y personas cercanas a ellos. Así, el objetivo de la presente revisión es analizar los impactos emocionales del cáncer infantil en la familia, discutiendo los diferentes sentimientos que despiertan en el núcleo familiar el proceso de tratamiento y eventual curación del cáncer infantil. Este estudio se estructura mediante una revisión integradora de la literatura, basada en la elaboración de la siguiente pregunta orientadora: “¿Cuáles son los impactos emocionales del cáncer infantil en la familia?"; la definición de bases de datos como PUBMED, SCIELO y VHL que sirvieron como instrumento de investigación; en base a los descriptores: Neoplasia, Niño, Familia, emociones y los criterios de elegibilidad para la revisión. En cuanto a los resultados, las familias reportaron numerosos impactos en la Familia como trastornos psicológicos, dificultades en las relaciones entre los padres y el cambio en la dinámica familiar debido al tratamiento de niños con cáncer pediátrico. Por tanto, entre las emociones negativas se destacaron el miedo, la irritabilidad, la ansiedad, la incertidumbre y la tristeza; en cuanto a los positivos, resiliencia, apoyo social, fe, esperanza, apertura, amor y positividad. El diagnóstico y posterior tratamiento del cáncer infantil es un momento muy delicado para toda la familia y el apoyo psicológico al niño y a la familia es fundamental para minimizar los efectos nocivos que provocan los malos sentimientos y ampliar el alcance de los buenos sentimentos.
\end{abstract}

Palabras clave: Neoplasma; Diagnóstico; Comportamiento infantil; Cuidadores; Emociones manifiestas.

\title{
1. Introdução
}

O câncer infantojuvenil, definido como câncer em crianças e adolescentes com idade entre 0-19 anos, corresponde a um grupo de várias doenças que têm em comum a proliferação descontrolada de células anormais e geralmente afeta as células do sistema sanguíneo e os tecidos de sustentação. Por serem predominantemente de natureza embrionária, tumores na criança e no adolescente são constituídos de células indiferenciadas (Feliciano, Santos \& Pombo-de-Oliveira, 2018).

As causas para o seu surgimento ainda são pouco exploradas e tem associação com a predisposição genética, hereditária, imunológica, exposição ambiental a agentes genotóxicos, radiações ionizantes, campos eletromagnéticos e outros. Apesar de ser considerado raro quando comparado com o câncer em adultos, pois afeta uma pequena proporção da carga global do câncer, tem incidência média estimada entre 0,5\% a 4,6\% de todos os tumores malignos (Feliciano et al., 2018).

Assim como nos países desenvolvidos, no Brasil, o câncer já representa a primeira causa de morte ( $8 \%$ do total) por doença entre crianças e adolescentes de 1 a 19 anos, configurando-se como um problema de saúde pública. Os tumores mais comuns nesta faixa etária são as leucemias, os que atingem o sistema nervoso central e os linfomas (Instituto Nacional do Câncer, 2016).

Nas últimas quatro décadas, o progresso no tratamento do câncer na infância e na adolescência foi extremamente significativo. Hoje, em torno de $80 \%$ de crianças e adolescentes acometidos podem ser curados, desde que diagnosticados precocemente e tratados em centros especializados (Instituto Nacional do Câncer, 2016).

Apesar dessa estimativa de cura, o câncer infantil gera impactos sentimentais profundos na vida de todos, sendo um grande indutor de sentimentos negativos na criança ou adolescente e nas pessoas próximas. Os primeiros enfrentam o medo e a incerteza do diagnóstico e os efeitos adversos do tratamento, implicando na perda da infância/adolescência, momento crucial na construção de relaçães com o próprio corpo e com o mundo externo, além da formação da sua personalidade que será base de todas as suas experiências futuras (Quintana, Wottrich, Camargo, Cherer \& Ries, 2011). Já os familiares ou cuidadores experimentam uma forte intensificação de seu papel e obrigações, diminuição do tempo disponível para suas próprias atividades, ansiedade, desgaste psicológico e interpessoal. (Alves et al., 2016)

Assim sendo, o objetivo deste trabalho é analisar os impactos emocionais do câncer infantil para a família, analisando os diversos sentimentos despertados no núcleo familiar pelo processo de tratamento e eventual cura do câncer infantojuvenil. 


\section{Metodologia}

O presente estudo está estruturado por meio de uma revisão integrativa da literatura, a qual permite uma atualização sobre a temática. Para a autora, as etapas metodológicas desta pesquisa abrangeram a elaboração da pergunta norteadora, a definição dos bancos de dados que serviram como instrumento de pesquisa, assim como a definição dos descritores, a definição dos critérios de elegibilidade, e a análise e inclusão dos estudos (Sousa, 2016).

A pergunta norteadora do estudo delineada foi a seguinte: "Quais os impactos emocionais do câncer infantil para a família?". Quanto aos procedimentos de coleta de dados, este foi realizado no período de maio a junho do ano de 2020 nas bases de dados: US National Library of Medicine National Institutes of Health (PUBMED), Scientific Electronic Library Online (SCIELO) e a Biblioteca Virtual em Saúde (BVS). Utilizaram-se os seguintes Descritores em Ciências da Saúde (DeCS), em inglês: Neoplasm, Child, Family e Emotions.

Quanto aos critérios de inclusão, consideraram-se estudos publicados em português, inglês, espanhol e francês entre os anos de 2011 a 2021. Foram excluídos artigos incompletos, estudos de revisão, como também teses, editoriais, estudos que não se relacionem aos DeCS, artigos repetidos nas diferentes bases de dados, além daqueles que não respondiam à pergunta norteadora do trabalho.

Conseguiu-se reunir 372 estudos relevantes nos bancos de dados. Destes, 196 foram excluídos com base nos critérios de exclusão, como ser dos anos anteriores à 2011 e não corresponder às línguas, português, inglês, espanhol e francês; 150 foram excluídos por estarem repetidos, com base no título e resumo e por não possuírem ligação com a pergunta norteadora do trabalho. Ao fim, reuniram-se 26 estudos que atenderam os critérios de elegibilidade definidos (Figura 1).

Figura 1: Fluxograma com a especificidade das buscas.

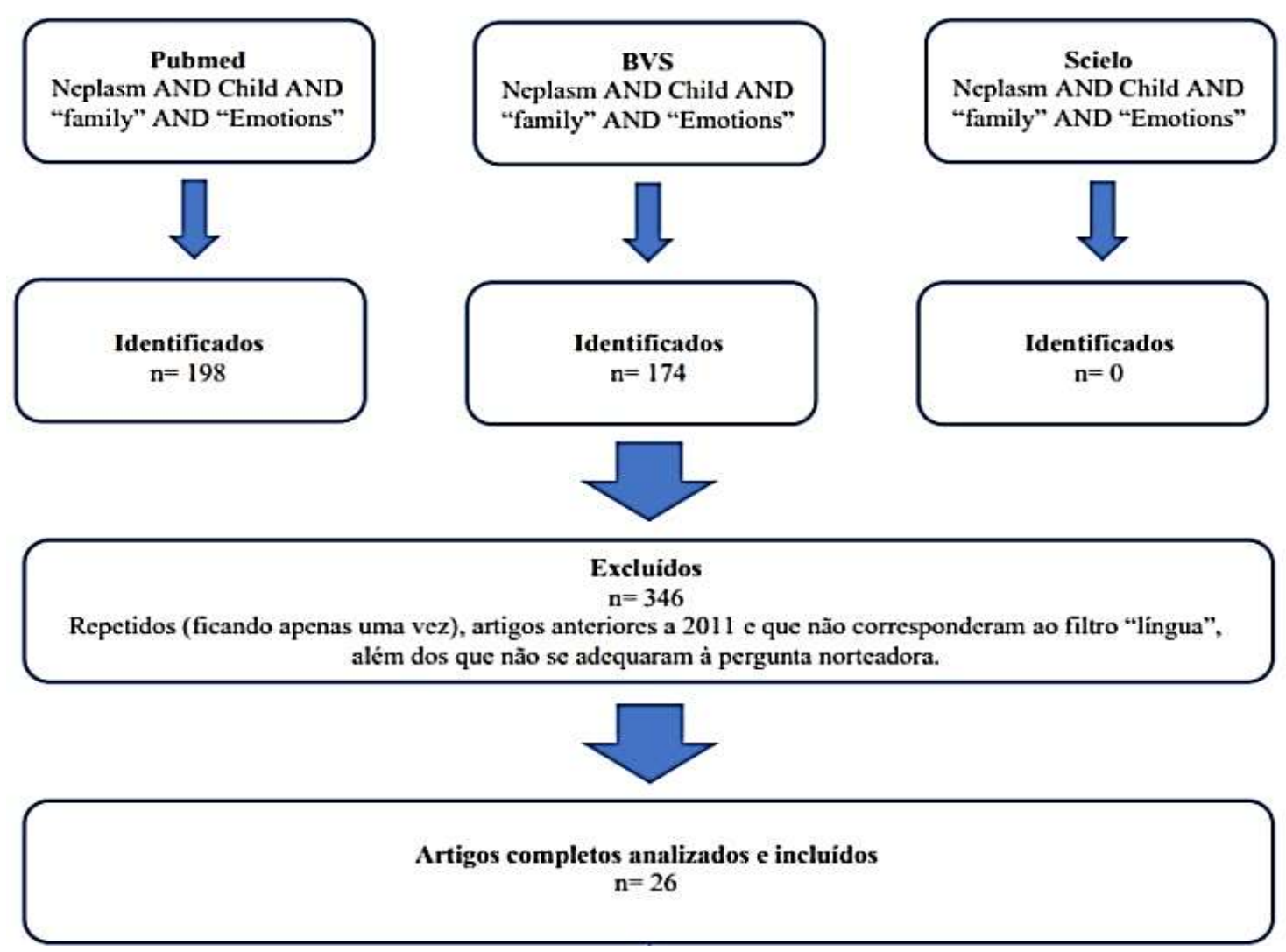

Fonte: Autores (2021). 
Dos estudos reunidos, sistematizaram-se informações quanto ao ano de publicação, o título do estudo, a base de dados e periódicos indexados, o idioma e o país, o método e os principais achados.

\section{Resultados}

Constata-se que o ano de prevalência das publicações foi o de 2019 (30,7\%; n=8). No que tange aos periódicos que serviram de veículos para a publicação dos artigos houve predominância do Journal of Pediatric Oncology Nursing (27,7\%; $\mathrm{n}=5)$, contudo, foram 18 diferentes periódicos. Sobre o idioma, o inglês foi o mais prevalente $(96,1 \%$; $n=25)$ e a base de dados que se destacou foi a US National Library of Medicine National Institutes of Health (PUBMED) (96,1\%; n=25). A respeito do Qualis Capes, a classificação A2 foi a mais constante $(42,3 \%$; $=11)$, já em relação ao país de publicação, foram encontrados 15 países distintos, com predominância dos Estados Unidos (40\%; n=6) (Quadro 1).

Quadro 1: Caracterização geral dos artigos selecionados para compor a RIL. Patos, 2021.

\begin{tabular}{|c|c|c|c|}
\hline Autores/Ano & Título do Artigo & Título do Periódico & $\begin{array}{l}\text { Qualis } \\
\text { Capes }\end{array}$ \\
\hline Buchbinder et al. (2017) & $\begin{array}{l}\text { Quality of Life Among Parents of Adolescent and } \\
\text { YoungAdult Brain Tumor Survivors }\end{array}$ & $\begin{array}{l}\text { Journal of Pediatric } \\
\text { Hematology/Oncology }\end{array}$ & $\mathrm{B} 2$ \\
\hline Cox (2016) & $\begin{array}{l}\text { Caregivers reflecting on the early days of childhood } \\
\text { cancer }\end{array}$ & European Journal of cancer care & $\mathrm{A} 3$ \\
\hline $\begin{array}{l}\text { D’Urso, } \\
\text { Mastroyannopoulou e Kirby } \\
\text { (2016) }\end{array}$ & $\begin{array}{l}\text { Experiences of posttraumatic growth in siblings of } \\
\text { children with cancer }\end{array}$ & $\begin{array}{l}\text { Clinical Child Psycholoy and } \\
\text { Psychiatry }\end{array}$ & A1 \\
\hline $\begin{array}{l}\text { Das, Khanna, Arora e } \\
\text { Agrawal (2019) }\end{array}$ & $\begin{array}{l}\text { Parents' acceptance and regret about end of life care for } \\
\text { children who died due to malignancy }\end{array}$ & Supportive Care in Cancer & $\mathrm{A} 2$ \\
\hline Doumit e Khoury (2017) & $\begin{array}{l}\text { Facilitating and hindering factors for coping with the } \\
\text { experience of having a child with cancer: A Lebanese } \\
\text { perspective }\end{array}$ & Journal of Psychosocial Oncology & B1 \\
\hline $\begin{array}{l}\text { Fortier, Wahi, Bruce, } \\
\text { Maurer e Stevenson (2013) }\end{array}$ & $\begin{array}{l}\text { Pain Management at Home in Children with Cancer: A } \\
\text { Daily Diary Study }\end{array}$ & Pediatric Blood \& Cancer & A3 \\
\hline Granek et al. (2012) & $\begin{array}{l}\text { Caregiving, single parents and cumulative stresses } \\
\text { when caring for a child with cancer }\end{array}$ & $\begin{array}{l}\text { Child: Care, Health and } \\
\text { Development }\end{array}$ & A2 \\
\hline $\begin{array}{l}\text { Hildenbrand, Clawson, } \\
\text { Alderfer e Marsac (2011) }\end{array}$ & $\begin{array}{l}\text { Coping with pediatric cancer: strategies employed by } \\
\text { children and their parents to manage cancer-related } \\
\text { stressors during treatment }\end{array}$ & $\begin{array}{l}\text { Journal of Pediatric Oncology } \\
\text { Nursing }\end{array}$ & A1 \\
\hline Hooghe et al. (2018) & $\begin{array}{l}\text { The child is our focus": On couple issues in child } \\
\text { oncology treatment }\end{array}$ & $\begin{array}{l}\text { Psycho-Oncology: Journal of the } \\
\text { Psychological, Social and } \\
\text { Behavioral Dimensions of Cancer }\end{array}$ & A1 \\
\hline Hooghe et al. (2020) & $\begin{array}{l}\text { "It's Hard to Talk When Your Child Has a Life } \\
\text { Threatening Illness": A Qualitative Study of Couples } \\
\text { Whose Child Is Diagnosed With Cancer }\end{array}$ & $\begin{array}{l}\text { Journal of pediatric oncology } \\
\text { nursing }\end{array}$ & A2 \\
\hline $\operatorname{Kim}(2017)$ & $\begin{array}{l}\text { Factors Influencing Posttraumatic Growth in Mothers } \\
\text { of Children With Cancer }\end{array}$ & $\begin{array}{l}\text { Journal of Pediatric Oncology } \\
\text { Nursing }\end{array}$ & A1 \\
\hline $\begin{array}{l}\text { Kostak, Semerci, Eren, } \\
\text { Avci e Savran (2019) }\end{array}$ & $\begin{array}{l}\text { Life experiences of adolescent with cancer in Turkey: a } \\
\text { phenomenological study }\end{array}$ & $\begin{array}{l}\text { Journal Of Pakistan Medical } \\
\text { Association }\end{array}$ & - \\
\hline Lazar e Musek (2020) & $\begin{array}{l}\text { Well-being in parents of children with cancer: The } \\
\text { impact of parental personality, coping, and the child's } \\
\text { quality of life }\end{array}$ & $\begin{array}{l}\text { Scandinavian Journal of } \\
\text { Psychology }\end{array}$ & $\mathrm{A} 2$ \\
\hline Long et al. (2013) & $\begin{array}{l}\text { Child-rearing in the context of childhood cancer: } \\
\text { perspectives of parents and professionals }\end{array}$ & Pediatric Blood \& Cancer & A3 \\
\hline Molinaro e Fletcher (2018) & $\begin{array}{l}\text { The Balancing Act: Mothers' Experiences of Providing } \\
\text { Care to Their Children With Cancer }\end{array}$ & $\begin{array}{l}\text { Journal of Pediatric Oncology } \\
\text { Nursing }\end{array}$ & A1 \\
\hline
\end{tabular}




\begin{tabular}{|c|c|c|c|}
\hline Moreira-Dias e Silva (2018) & $\begin{array}{l}\text { A Utilização do Brinquedo durante o Tratamento de } \\
\text { Crianças com Câncer: Percepções da Equipe } \\
\text { Multidisciplinar }\end{array}$ & $\begin{array}{l}\text { Revista Brasileira de } \\
\text { Cancerologia }\end{array}$ & B2 \\
\hline $\begin{array}{l}\text { Nolbris, Enskär e Hellström } \\
\text { (2014) }\end{array}$ & $\begin{array}{l}\text { Grief related to the experience of being the sibling of a } \\
\text { child with cancer }\end{array}$ & Cancer Nursing & A2 \\
\hline $\begin{array}{l}\text { Peterson, Chung e Barrera } \\
\text { (2019) }\end{array}$ & $\begin{array}{l}\text { Emotional symptoms and family functioning in } \\
\text { caregivers of children with newly diagnosed } \\
\text { leukemia/lymphomas and solid tumors: Short-term } \\
\text { changes and related demographic factors }\end{array}$ & Wiley Online Library & B1 \\
\hline $\begin{array}{l}\text { Popp, Conway e Pantaleao } \\
\text { (2015) }\end{array}$ & $\begin{array}{l}\text { Parents' Experience With Their Child's Cancer } \\
\text { Diagnosis: Do Hopefulness, Family Functioning, and } \\
\text { Perceptions of Care Matter? }\end{array}$ & $\begin{array}{l}\text { Journal of Pediatric Oncology } \\
\text { nursing }\end{array}$ & A1 \\
\hline Rensen et al. (2019a) & $\begin{array}{l}\text { Determinants of health-related quality of life proxy } \\
\text { rating disagreement between caregivers of children } \\
\text { with cancer }\end{array}$ & Quality of life research & A2 \\
\hline Rensen et al. (2019b) & $\begin{array}{l}\text { Gender-specific differences in parental health-related } \\
\text { quality of life in childhood cancer }\end{array}$ & Wiley Online Library & B1 \\
\hline $\begin{array}{l}\text { Santos, Wiegand, Sá, Misko } \\
\text { e Szylit (2019) }\end{array}$ & $\begin{array}{l}\text { From hospitalization to grief: meanings parents assign } \\
\text { to their relationships with pediatric oncology } \\
\text { professional }\end{array}$ & $\begin{array}{l}\text { Revista da escola de enfermagem } \\
\text { da USP }\end{array}$ & A2 \\
\hline Shi et al. (2016) & $\begin{array}{l}\text { Prevalence and predictors of posttraumatic stress } \\
\text { symptoms in parents of children with ongoing } \\
\text { treatment for cancer in South China: a multi-centered } \\
\text { cross-sectional study }\end{array}$ & Supportive Care in Cancer & A2 \\
\hline Sisk, Kang e Mack (2019) & $\begin{array}{l}\text { The evolution of regret: decision-making for parents of } \\
\text { children with cancer }\end{array}$ & Supportive Care in Cancer & $\mathrm{A} 2$ \\
\hline $\begin{array}{l}\text { Van Schoors, Mol, } \\
\text { Verhofstadt, Goubert e Van } \\
\text { parys (2019) }\end{array}$ & $\begin{array}{l}\text { The family practice of support-giving after a pediatric } \\
\text { cancer diagnosis: A multi-family member interview } \\
\text { analysis }\end{array}$ & $\begin{array}{l}\text { European Journal of Oncology } \\
\text { Nursing }\end{array}$ & B1 \\
\hline $\begin{array}{l}\text { Watanabe, Nunes, Abreu } \\
\text { (2013) }\end{array}$ & $\begin{array}{l}\text { Japanese parents' perception of disclosing the diagnosis } \\
\text { of cancer to their children }\end{array}$ & $\begin{array}{l}\text { Clinical Child Psychology and } \\
\text { Psychiatry }\end{array}$ & A1 \\
\hline
\end{tabular}

Fonte: Autores.

Com relação ao enquadramento dos estudos, encontraram-se sete tipos diferentes de pesquisas, sendo a maioria qualitativa $(69,2 \% ; n=18)$. A respeito da população-alvo, notaram-se quatro grupos distintos, com os parentes de crianças com câncer em destaque $(84,6 \% ; n=22)$. Constatou-se que a média do tamanho amostral das pesquisas foi de aproximadamente 94 participantes, sendo assim, nove (34,6\%) trabalhos apresentaram números superiores (Quadro 2).

Quadro 2: Caracterização metodológica dos artigos selecionados para compor a RIL. Patos, 2021.

\begin{tabular}{|c|c|c|c|}
\hline Autores/Ano & Tipo de pesquisa & População-alvo & Tamanho amostral \\
\hline $\begin{array}{l}\text { Buchbinder et al. } \\
\text { (2017) }\end{array}$ & Pesquisa qualitativa & $\begin{array}{l}\text { Pais de adolescentes ou jovens adultos com mais de } 2 \\
\text { anos de diagnóstico e falantes de língua inglesa ou } \\
\text { espanhola. }\end{array}$ & $\begin{array}{l}\text { Amostra inicial: } 49 \text { famílias. } \\
\text { Amostra final: } 28 \text { famílias }\end{array}$ \\
\hline Cox (2016) & Pesquisa qualitativa & $\begin{array}{l}\text { Adolescente / Adulto / Criança / Criança, pré-escolar } \\
\text { / Feminino / Humanos / Lactente / Masculino / Meia- } \\
\text { Idade / Recém-Nascido }\end{array}$ & 38 participantes \\
\hline $\begin{array}{l}\text { D'Urso et al. } \\
(2016)\end{array}$ & Pesquisa qualitativa & $\begin{array}{l}\text { Adolescentes entre } 12 \text { e } 18 \text { anos cujos irmão ou irmã } \\
\text { de até } 18 \text { anos que tenha tido câncer }\end{array}$ & $\begin{array}{l}\text { Amostra inicial: } 7 \text { participantes. } \\
\text { Amostra final: } \\
\text { participantes }\end{array}$ \\
\hline Das et al. (2019) & $\begin{array}{l}\text { Estudo qualitativo } \\
\text { observacional }\end{array}$ & $\begin{array}{l}\text { Pais de pacientes pediátricos com malignidade } \\
\text { curáveis que morreram durante os anos de 2016-2018 }\end{array}$ & 26 famílias \\
\hline $\begin{array}{l}\text { Doumit e Khoury } \\
\text { (2017) }\end{array}$ & $\begin{array}{l}\text { Análise de dados } \\
\text { textuais }\end{array}$ & $\begin{array}{l}\text { Pais libaneses que têm filhos com câncer, enfermeiras } \\
\text { e médicos. }\end{array}$ & 12 pais \\
\hline
\end{tabular}




\begin{tabular}{|c|c|c|c|}
\hline Fortier et al. (2013) & Pesquisa qualitativa & $\begin{array}{l}\text { Adolescente / Criança / Feminino / Humanos / } \\
\text { Masculino }\end{array}$ & 90 participantes \\
\hline $\begin{array}{l}\text { Granek et al. } \\
\text { (2012) }\end{array}$ & Pesquisa qualitativa & $\begin{array}{l}\text { Cuidadores primários e crianças em tratamento contra } \\
\text { o câncer entre } 6 \text { e } 12 \text { anos, com diagnóstico entre } 1 \\
\text { semana e } 3 \text { anos e meio. }\end{array}$ & $\begin{array}{l}\text { Amostra inicial e final: } 15 \\
\text { cuidadores de crianças com câncer, } \\
\text { além das próprias crianças. }\end{array}$ \\
\hline $\begin{array}{l}\text { Hildenbrand } \text { et al. } \\
\text { (2011) }\end{array}$ & $\begin{array}{l}\text { Pesquisa qualitativa } \\
\text { consensual }\end{array}$ & Pais cujos filhos estavam em tratamento de câncer & 16 famílias \\
\hline $\begin{array}{l}\text { Hooghe et al. } \\
\text { (2018) }\end{array}$ & Pesquisa qualitativa & Pais cujos filho estava em tratamento de câncer & 16 pais \\
\hline $\begin{array}{l}\text { Hooghe et al. } \\
\text { (2020) }\end{array}$ & Pesquisa qualitativa & $\begin{array}{l}\text { Irmãos com idade entre } 8 \text { a } 25 \text { anos de crianças } \\
\text { tratadas contra o câncer. }\end{array}$ & $\begin{array}{l}\text { Amostra inicial e final: } 29 \\
\text { participantes. }\end{array}$ \\
\hline $\operatorname{Kim}(2017)$ & Relato de caso & Feminino/ humanos & 222 participantes \\
\hline Kostak et al. (2019) & $\begin{array}{l}\text { Estudo qualitativo } \\
\text { fenomenológico }\end{array}$ & Profissionais de saúde, especialmente enfermeiros. & 12 adolescentes \\
\hline $\begin{array}{l}\text { Lazar e Musek } \\
(2020)\end{array}$ & Pesquisa qualitativa & Pais/mães solteiros & $\begin{array}{l}\text { Amostra inicial: } 29 \text { participantes. } \\
\text { Amostra final: } 19 \text { participantes }\end{array}$ \\
\hline Long et al. (2013) & Estudo multivariado & $\begin{array}{l}\text { Pais de crianças, tratados de câncer no Departamento } \\
\text { de Oncologia e Hematologia, Divisão de Pediatria, } \\
\text { University Medical Center Ljubljana, Eslovênia. }\end{array}$ & 99 pais (63 mães e 36 pais) \\
\hline $\begin{array}{l}\text { Molinaro e Fletcher } \\
(2018)\end{array}$ & $\begin{array}{l}\text { Estudo qualitativo e } \\
\text { quantitativo }\end{array}$ & Crianças com diagnóstico de câncer. & 345 participantes \\
\hline $\begin{array}{l}\text { Moreira- Dias e } \\
\text { Silva (2018) }\end{array}$ & Estudo qualitativo & Feminino / Humanos / Masculino & 10 participantes. \\
\hline $\begin{array}{l}\text { Nolbris et al. } \\
\text { (2014) }\end{array}$ & Pesquisa qualitativa & $\begin{array}{l}\text { Mulher de no mínimo } 16 \text { anos e parente de uma } \\
\text { criança com menos de } 17 \text { anos que foi ou está sendo } \\
\text { tratada contra o câncer nos últimos } 5 \text { anos/ } \\
\text { profissional de saúde que trabalha diretamente com } \\
\text { famílias cujos filhos tiveram câncer. }\end{array}$ & $\begin{array}{l}\text { Amostra inicial: } \\
14 \text { mães e } 3 \text { cuidadoras. } \\
\text { Amostra final: } \\
9 \text { mães e } 3 \text { cuidadoras. }\end{array}$ \\
\hline $\begin{array}{l}\text { Peterson et al. } \\
(2019)\end{array}$ & Pesquisa qualitativa & $\begin{array}{l}\text { Cuidadores de crianças com um diagnóstico recente } \\
\text { de leucemia / linfoma ou tumor sólido. }\end{array}$ & 122 Cuidadores \\
\hline Popp et al. (2015) & $\begin{array}{l}\text { Estudo de } \\
\text { experiência inicial }\end{array}$ & Pais que têm um filho com diagnóstico de câncer & 50 pais \\
\hline $\begin{array}{l}\text { Rensen et al. } \\
\text { (2019a) }\end{array}$ & $\begin{array}{l}\text { Estudo } \\
\text { multicêntrico } \\
\text { transversal }\end{array}$ & $\begin{array}{l}\text { Pais com uma criança diagnosticada com câncer ou } \\
\text { qualquer tipo de tumor cerebral. }\end{array}$ & 120 pais \\
\hline $\begin{array}{l}\text { Rensen et al. } \\
\text { (2019b) }\end{array}$ & Estudo transversal & Pais de criança com câncer & 202 mães, 150 pais \\
\hline Santos et al. (2019) & $\begin{array}{l}\text { Estudo qualitativo- } \\
\text { interpretativo }\end{array}$ & Profissionais de saúde & 42 famílias \\
\hline Shi et al. (2016) & Pesquisa transversal & Pais de crianças com câncer. & 279 pais \\
\hline Sisk et al. (2019) & Estudo prospectivo & $\begin{array}{l}\text { Pais de crianças com câncer em dois hospitais } \\
\text { pediátricos acadêmicos }\end{array}$ & 158 participantes \\
\hline $\begin{array}{l}\text { Van Schoors et al. } \\
(2019)\end{array}$ & Análise qualitativa & Famílias de crianças com câncer pediátrico & 173 pais, 55 casais e 27 irmãos. \\
\hline $\begin{array}{l}\text { Watanabe et al. } \\
\text { (2013) }\end{array}$ & Pesquisa qualitativa & $\begin{array}{l}\text { Adolescente / Criança / Feminino / Humanos / } \\
\text { Masculino }\end{array}$ & 58 participantes \\
\hline
\end{tabular}

Fonte: Autores.

Constatou-se que os trabalhos apresentaram como principais objetivos: estudar e compreender os fatores psicossociais envolvendo a família no tratamento da criança com câncer; descrever e entender as emoções e os sintomas psicológicos apresentados por pais e filhos com câncer pediátrico $(61,5 \%$; n=16). Com relação aos resultados, as famílias relataram inúmeros impactos na qualidade de vida do núcleo familiar, vários distúrbios psicológicos, dificuldades nas relações entre os pais e a mudança na dinâmica familiar em virtude do tratamento da criança com câncer pediátrico $(69,2 \%$; $n=18)$. A respeito 
da categorização dos estudos, notou-se que o mais recorrente foi: emoções negativas ( $\mathrm{n}=37)$ - medo (38\%; $n=14)$, irritabilidade (24\%; $n=9)$, ansiedade, incerteza e tristeza (esses três últimos apresentaram igual porcentagem, 19\%; n=7) e emoções positivas $(n=29)$ - resiliência $(21 \% ; n=6)$, suporte social, fé e esperança (apresentaram igual porcentagem, 10\%; n=3) e abertura, amor e positividade (registraram o mesmo percentual, 7\%; n=2). (Quadro 3).

Quadro 3: Categorização dos achados da RIL. Patos, 2021.

\begin{tabular}{|c|c|c|c|c|}
\hline Categorias & Subcategorias & Autores & $\mathbf{n}$ & $\%$ \\
\hline \multirow{26}{*}{$\begin{array}{l}\text { Emoções } \\
\text { Negativas }\end{array}$} & Angústias & $\begin{array}{l}\text { Doumit e Khoury (2017); Peterson et al. (2019); Rensen } \text { et al. } \\
\text { (2019a); Rensen } \text { et al. (2019b); Santos } \text { et al. (2019) }\end{array}$ & 5 & $14 \%$ \\
\hline & Ansiedade & $\begin{array}{l}\text { Doumit e Khoury (2017); Hildenbrand et al. (2011); Kostak et } \\
\text { al. (2019); Lazar e Musek (2020); Moreira-Dias e Silva (2018); } \\
\text { Nolbris et al. (2014); Peterson } \text { et al. (2019) }\end{array}$ & 7 & $19 \%$ \\
\hline & Arrependimento & Das et al. (2019); Sisk et al. (2019) & 2 & $5 \%$ \\
\hline & Baixa autoestima & Lazar e Musek (2020) & 1 & $3 \%$ \\
\hline & $\begin{array}{l}\text { Ceticismo/ Desesperança/ } \\
\text { Pessimismo }\end{array}$ & $\begin{array}{l}\text { D’Urso et al. (2016); Granek et al. (2012); Hildenbrand et al. } \\
\text { (2011) }\end{array}$ & 3 & $8 \%$ \\
\hline & Choque & D’Urso et al. (2016); Kostak et al. (2019); Popp et al. (2015) & 3 & $8 \%$ \\
\hline & $\begin{array}{l}\text { Conflitos, discordância entre os pais } \\
\text { e/ou mau funcionamento familiar }\end{array}$ & $\begin{array}{l}\text { Hooghe } \text { et al. (2018); Peterson et al. (2019); Rensen et al. } \\
\text { (2019a); Shi } \text { et al. (2016) }\end{array}$ & 4 & $11 \%$ \\
\hline & Culpa/Auto culpa & $\begin{array}{l}\text { D’Urso et al. (2016); Lazar e Musek (2020); Long et al. } \\
\text { (2013); Sisk et al. (2019) }\end{array}$ & 4 & $11 \%$ \\
\hline & Decepção & Kostak et al. (2019) & 1 & $3 \%$ \\
\hline & Depressão & $\begin{array}{l}\text { Hooghe et al. (2020); Lazar e Musek (2020); Molinaro e } \\
\text { Fletcher (2018); Peterson } \text { et al. (2019); Rensen } \text { et al. (2019b); } \\
\text { Shi } \text { et al. (2016) }\end{array}$ & 6 & $16 \%$ \\
\hline & Desamparo & Long et al (2013) & 1 & $3 \%$ \\
\hline & $\begin{array}{l}\text { Dificuldade em expressar seus } \\
\text { sentimentos }\end{array}$ & Peterson et al. (2019) & 1 & $3 \%$ \\
\hline & Dor & Fortier et al. (2013) & 1 & $3 \%$ \\
\hline & Dúvidas & Santos et al. (2019) & 1 & $3 \%$ \\
\hline & Estresse & $\begin{array}{l}\text { Doumit e Khoury (2017); Kostak et al. (2019); Molinaro e } \\
\text { Fletcher (2018); Shi et al. (2016) }\end{array}$ & 4 & $11 \%$ \\
\hline & $\begin{array}{l}\text { Falta de acesso aos profissionais de } \\
\text { saúde e medicação para dor }\end{array}$ & Das et al. (2019) & 1 & $3 \%$ \\
\hline & $\begin{array}{l}\text { Falta de apoio social/ Diminuição de } \\
\text { apoio }\end{array}$ & Peterson et al. (2019); Rensen et al. (2019b) & 2 & $5 \%$ \\
\hline & Frustração & Granek et al. (2012) & 1 & $3 \%$ \\
\hline & Incerteza & $\begin{array}{l}\text { Buchbinder } \text { et al. (2017); D'Urso et al. (2016); Moreira-Dias e } \\
\text { Silva (2018); Fortier } \text { et al. (2013); Kostak et al. (2019); } \\
\text { Molinaro e Fletcher (2018); Schoors } \text { et al. (2019); Watanabe } \text { et } \\
\text { al. (2013) }\end{array}$ & 7 & $19 \%$ \\
\hline & Inveja & D’Urso et al. (2016) & 1 & $3 \%$ \\
\hline & Mágoas & Santos et al. (2019) & 1 & $3 \%$ \\
\hline & Mau funcionamento familiar & Hooghe et al. (2018); Shi et al. (2016) & 2 & $5 \%$ \\
\hline & Medo/Medo de Morte & $\begin{array}{l}\text { Cox (2016); D’Urso et al. (2016); Moreira-Dias e Silva (2018); } \\
\text { Doumit e Khoury (2017); Fortier et al. (2013); Hildenbrand } \text { et } \\
\text { al. (2011); Hooghe et al. (2020); Kostak et al. (2019); Das et } \\
\text { al. (2019); Lazar e Musek (2020); Nolbris et al. (2014); } \\
\text { Schoors } \text { et al. (2019); Sisk et al. (2019); Watanabe } \text { et al. (2013) }\end{array}$ & 14 & $38 \%$ \\
\hline & Nervosismo & Doumit e Khoury (2017); Hildenbrand et al. (2011) & 2 & $5 \%$ \\
\hline & Opressão & Popp et al. (2015) & 1 & $3 \%$ \\
\hline & Perda/ Sentimento de perda & Cox (2016); Das et al. (2019) & 2 & $5 \%$ \\
\hline
\end{tabular}


Research, Society and Development, v. 10, n. 10, e56101017931, 2021

(CC BY 4.0) | ISSN 2525-3409 | DOI: http://dx.doi.org/10.33448/rsd-v10i10.17931

\begin{tabular}{|c|c|c|c|c|}
\hline & Preocupação & D’Urso et al. (2016); Nolbris et al. (2014); Popp et al. (2015) & 3 & $8 \%$ \\
\hline & $\begin{array}{l}\text { Problemas emocionais/ instabilidade } \\
\text { emocional/ Diminuição do bem estar } \\
\text { psicológico }\end{array}$ & $\begin{array}{l}\text { Granek et al. (2012); Lazar e Musek (2020); Rensen et al. } \\
\text { (2019b) }\end{array}$ & 3 & $8 \%$ \\
\hline & Raiva/Irritabilidade & $\begin{array}{l}\text { Buchbinder } \text { et al. (2017); Cox (2016); D’Urso et al. (2016); } \\
\text { Granek et al. (2012); Hooghe } \text { et al. (2020); Das et al. (2019); } \\
\text { Lazar e Musek (2020); Peterson et al. (2019); Popp et al. } \\
\text { (2015) }\end{array}$ & 9 & $24 \%$ \\
\hline & Rivalidade & Doumit e Khoury (2017) & 1 & $3 \%$ \\
\hline & Separação familiar & Schoors et al. (2019) & 1 & $3 \%$ \\
\hline & Sofrimento & $\begin{array}{l}\text { Buchbinder et al. (2017); Granek et al. (2012); Hooghe et al. } \\
\text { (2018) }\end{array}$ & 3 & $8 \%$ \\
\hline & Solidão & Kunal Das et al. (2019) & 1 & $3 \%$ \\
\hline & Tédio & Kunal Das et al. (2019) & 1 & $3 \%$ \\
\hline & Tensão & Doumit e Khoury (2017) & 1 & $3 \%$ \\
\hline & Tristeza & $\begin{array}{l}\text { Buchbinder et al. (2017); D’Urso et al. (2016); Granek et al. } \\
\text { (2012); Hildenbrand et al. (2011); Hooghe et al. (2020); } \\
\text { Nolbris et al. (2014); Popp et al. (2015) }\end{array}$ & 7 & $19 \%$ \\
\hline \multirow{26}{*}{$\begin{array}{l}\text { Emoções } \\
\text { Positivas }\end{array}$} & Abertura & Hooghe et al. (2020); Lazar e Musek (2020) & 2 & $7 \%$ \\
\hline & Aceitação & Moreira-Dias e Silva (2018) & 1 & $3 \%$ \\
\hline & Afeto & Lazar e Musek (2020) & 1 & $3 \%$ \\
\hline & Alegria & Moreira-Dias e Silva (2018) & 1 & $3 \%$ \\
\hline & Amor/Amabilidade & Lazar e Musek (2020); Schoors et al. (2019) & 2 & $7 \%$ \\
\hline & $\begin{array}{l}\text { Apoio/Apoio emocional/Suporte } \\
\text { Social }\end{array}$ & $\begin{array}{l}\text { Doumit e Khoury (2017); Santos et al. (2019); Schoors et al. } \\
\text { (2019) }\end{array}$ & 3 & $10 \%$ \\
\hline & Autoconfiança & D’Urso et al. (2016) & 1 & $3 \%$ \\
\hline & Baixo neuroticismo & Lazar e Musek (2020) & 1 & $3 \%$ \\
\hline & Carinho & Hooghe et al. (2018) & 1 & $3 \%$ \\
\hline & $\begin{array}{l}\text { Companheirismo, parceria e } \\
\text { satisfação conjugal }\end{array}$ & Hooghe et al. (2018); Popp et al. (2015); Schoors et al. (2019) & 3 & $10 \%$ \\
\hline & Compromisso & Hooghe et al. (2018) & 1 & $3 \%$ \\
\hline & Consciência & Lazar e Musek (2020) & 1 & $3 \%$ \\
\hline & Consolo & Schoors et al. (2019) & 1 & $3 \%$ \\
\hline & Disponibilidade & Schoors et al. (2019) & 1 & $3 \%$ \\
\hline & $\begin{array}{l}\text { Efeito positivo da religião/ Crenças } \\
\text { fundamentais/ Fé }\end{array}$ & Doumit e Khoury (2017); Kim (2017); Shi et al. (2016) & 3 & $10 \%$ \\
\hline & Esperança & Cox (2016); Hildenbrand et al. (2011); Popp et al. (2015) & 3 & $10 \%$ \\
\hline & Estabilidade emocional & Lazar e Musek (2020) & 1 & $3 \%$ \\
\hline & Experiência de aprendizado & Hildenbrand et al. (2011) & 1 & $3 \%$ \\
\hline & Extroversão & Lazar e Musek (2020) & 1 & $3 \%$ \\
\hline & Força & Granek et al. (2012) & 1 & $3 \%$ \\
\hline & Melhor comunicação & Watanabe et al. (2013) & 1 & $3 \%$ \\
\hline & $\begin{array}{l}\text { Otimismo/ } \\
\text { postividade }\end{array}$ & Granek et al. (2012); Kim (2017); Lazar e Musek (2020) & 3 & $10 \%$ \\
\hline & Proteção & Santos et al. (2019) & 1 & $3 \%$ \\
\hline & Resiliência & $\begin{array}{l}\text { Moreira-Dias e Silva (2018); Granek et al. (2012); Hooghe et } \\
\text { al. (2018); Long et al. (2013); Shi et al. (2016); Watanabe } \text { et al. } \\
\text { (2013) }\end{array}$ & 6 & $21 \%$ \\
\hline & Resolução de conflitos & Hooghe et al. (2018) & 1 & $3 \%$ \\
\hline & Respeito & Popp et al. (2015) & 1 & $3 \%$ \\
\hline
\end{tabular}

Fonte: Autores. 


\section{Discussão}

Vivenciar uma doença grave, como o câncer infantil, desperta uma cascata de emoções, especialmente as negativas (Hildenbrand et al., 2011; Fortier et al., 2013; Popp et al., 2015; Cox, 2016; D'urso et al., 2016; Buchbinder et al., 2017; Doumit \& Khoury, 2017; Moreira-Dias \& Silva, 2018; Kostak et al., 2019; Das et al., 2019; Peterson et al., 2019; Rensen et al., 2019a; Rensen et al., 2019b; Schoors et al., 2019; Hooghe et al., 2020; Lazar \& Musek, 2020.). Entre essas se destacaram, principalmente, o medo como o mais relevante $(38 \% ; n=14)$, seguido de irritabilidade (24\%; $n=9)$, ansiedade, incerteza e tristeza (esses três últimos apresentaram igual porcentagem, 19\%; $\mathrm{n}=7$ ).

Quanto às emoções positivas (Granek et al., 2012; Long et al., 2013; Watanabe et al., 2013; Popp et al., 2015; Shi et al., 2016; Santos et al., 2019; Schoors et al., 2019; Hooghe et al., 2020;), houve um destaque para resiliência (21\%; n=6), seguido de suporte social, fé, otimismo, companheirismo e esperança (apresentaram igual porcentagem, 10\%; $n=3$ ) e abertura e amor (registraram o mesmo percentual, $7 \% ; n=2$ ).

Sobre as emoções negativas, as mais fortes e importantes, foram as variáveis psicossociais, os problemas emocionais e a percepção da falta de apoio social a algumas famílias (Rensen et al., 2019b). É mister destacar o medo e os outros sentimentos decorrentes dele, como a angústia e a tristeza diante do sofrimento do paciente. A fragilidade inerente à condição infantil desses pacientes é explicada pelo fato de que, frequentemente, eles não conseguem se expressar com clareza, e dessa forma definir a localização e amplitude dos sintomas, principalmente da dor (Castro, 2010).

São inquestionáveis os inúmeros impactos psicológicos causados por diagnóstico de câncer pediátrico tanto na criança, quanto nas pessoas que estão ao seu redor. Pôde-se observar que ao descobrir a doença as crianças experimentaram choque, tristeza, medo, ansiedade, curiosidade e incerteza, além disso, com o passar do tempo, devido às mudanças físicas, sentiram que tinham se tornado outra pessoa, se achavam "estranhos" e não queriam acreditar que o que estavam passando era verdade (Kostak et al., 2019).

Pode-se notar, também, que alguns sentimentos nas relações familiares foram vistos pelas crianças diagnosticadas, como sentir falta de seus irmãos e pais, culpa, raiva e ciúme. Já os irmãos ou irmãs do paciente se sentiam negligenciados e apreensivos sobre o sucesso do tratamento e com medo da perda de um ente querido. Dessa forma, o câncer afetou diretamente as relações familiares negativamente (Kostak et al., 2019).

Na mesma linha, o diagnóstico e o tratamento da criança implicam na modificação abrupta da dinâmica familiar, levando tanto a criança quanto o cuidador primário a se ausentar do convívio dos outros membros, como o marido e os outros filhos, fato que é gerador de sentimentos como solidão e carência no parceiro; e nos filhos, raiva e inveja (Granek et al., 2012; D'Urso et al., 2016)

Importante destacar o nível de correlação entre essas emoções experimentadas pelos envolvidos diretamente no tratamento do câncer, posto que não só o sofrimento enfrentado pelas crianças afeta a relação dos pais, como também as relações dos pais e a maneira deles enfrentarem o tratamento influenciam diretamente tanto o bem estar da criança durante o tratamento, como também muitas das vezes melhoram a eficácia do tratamento (Lazar \& Musek, 2020).

Evidencia-se que os sintomas emocionais do cuidador variam de acordo com o tipo de câncer que foi diagnosticado. Crianças com leucemia/linfoma tiveram seus pais com níveis muito altos de ansiedade e sofrimento significativo no momento do diagnóstico, já os que tinham tumores sólidos, tiveram seus pais com ansiedade limítrofe e sofrimento no momento e até seis meses depois do diagnóstico (Peterson et al., 2019).

Algumas reações não resolvidas foram observadas nos pais que interferem diretamente nas interações pais e filhos, essas reações foram representadas pelas falas: "Eu não conseguia nem olhar para ele ou estar perto dele após o diagnóstico", "Eu não senti nada sobre o diagnóstico", "Eu me pergunto se foi alguma coisa que fiz durante a gravidez, foi algo que dei a ela, 
eu me pergunto por que o tempo todo" essas frases mostram um desligamento emocional e a busca contínua para entender o porquê de estar acontecendo (Popp et al., 2015, p. 5).

Entre as emoções positivas, o otimismo teve significativo resultado com a ruminação deliberada no crescimento póstraumático (PTC), assim como os efeitos diretos e indiretos para o suporte pós-traumático (Kim, 2016). A ruminação psicológica é explicada como os pensamentos negativos e recorrentes. Apesar de todas as pessoas ruminarem, nem toda ruminação é desestrutural.

As pessoas que ruminam habitualmente em resposta a um sofrimento, tendem a exibir dependência em suas experiências afetivas negativas, criar neles mesmos uma perturbação emocional.

A ruminação é prejudicial quando associado a outros pensamentos disfóricos como os pensamentos que trazem prejuízo ao funcionamento psíquico de um indivíduo, pois potencializa os indivíduos a perceber negativamente o ambiente e os acontecimentos que os rodeiam e, assim a partir destas percepções, como a modificação de papéis, ainda que possa promover sentimentos para crenças positivas, pode também inovar ao provocar novos padrões de comportamento, sendo que normalmente o restabelecimento da criança doente é o fator que mais mobiliza a família, o que faz se necessário o apoio social como influência diretamente para os transtornos pós-traumáticos (Quintana et al., 2011).

Percebe-se, também, que durante o tratamento das crianças, devido a mudanças na rotina do casal ocorram conflitos matrimoniais e consequências para o relacionamento sexual do casal devido aos vínculos afetivos que unem os membros da família, assim como a organização das tarefas nas rotinas, em virtude das projeções esperadas pelo cuidador principal sobre o outro, em compreender sua posição de dedicação total ao filho. Assim, a desestruturação familiar inicia pelo desequilíbrio da relação na unidade conjugal, através do rompimento dos laços afetivos entre o casal a fim de dedicarem-se exclusivamente ao filho, por serem tomados por sentimentos negativos e pelo medo que cerca as consequências do câncer.

Quanto às formas de amparo para as mães, a mais significante foi o apoio familiar, sugerindo que os membros da família são fontes essenciais de apoio para os pais de crianças com câncer. Apesar de tentarem um significado positivo, sendo otimistas, como por exemplo: utilizar a doença como forma de aprendizado, foi notório o desenvolvimento de transtornos póstraumáticos (TPT) ou sintomas indicativos de câncer relacionado ao TPT. Apesar dos esforços dos pais para se manterem firmes e com bons pensamentos ao redor dos problemas relacionados à doença, a maioria acaba os absorvendo.

Ademais, o sofrimento da criança e o agravamento do seu estado de saúde levam os cuidadores primários, muitas vezes as mães, ao desespero. Fazendo com que elas peçam para trocar de lugar com ele, se questionando frequentemente por que eles e não elas (Hildenbrand et al., 2011; D’Urso et al., 2016). Notou-se que existem diferenças significantes entre mães e pais, sendo as mães mais vulneráveis a sofrimentos emocionais (Rensen et al., 2019b).

O desejo de reverter para si à dor pela qual seus filhos estejam passando é mobilizador de extrema comoção no núcleo familiar, muitas vezes causadores de rupturas na relação conjugal ou na relação entre os irmãos (Nolbris et al., 2014). Nesse sentido, questões que ficam em aberto ou mal resolvidas acerca do tratamento ou do cuidado resultam em mágoas, dúvidas e angústias em relação à doença do filho e, se não forem trabalhadas, permanecem inalteradas, mesmo anos após a morte ou o tratamento da criança (Santos et al., 2019).

Já o cuidador primário se sente excluído, por ter sido o membro familiar que mais abdicou de toda sua rotina e/ou trabalho para estar presente no tratamento (Molinaro e Fletcher, 2018). De repente, ele se vê estranho nesse mundo novo, desconhecido, diferente. Ocorre à perda de sua liberdade, de seu querer, deixa de ser si mesmo para confundir-se com a doença, passa a ser inautêntico, impessoal. A esse fenômeno Heidegger (2002) se refere como a estranheza na angústia.

Ao se deparar com o câncer, uma doença crônica de progressão frequentemente silenciosa, cuja eficácia do tratamento ainda não é totalmente esclarecida faz com que algumas mães pensem continuamente na morte do próprio filho (Hildenbrand 
et al., 2011) reforçando o estigma do câncer como doença terminal e a, consequente, descrença no tratamento (Granek et al., 2012).

Da mesma forma, os pacientes que acabam descobrindo um câncer vivenciam as mais variadas sensações, principalmente quando assimila esse achado com a finitude, independente da possibilidade de tratamento, fazendo uma quebra com a própria vida. Gerando, muitas vezes, a não aceitação da condição pela qual está passando (Dib, Gomes, Ramos, França \& Marques, 2020).

Ademais, apesar da prevalência geral dos sentimentos de medo, angústia e tristeza, destaca-se também a questão do arrependimento, principalmente quando se fala nos meses terminais. Esse arrependimento se apresenta por diversos fatores, mas entre eles os prevalentes se dão devido à sensação de negligência durante alguma etapa do tratamento (aquela sensação de que poderia ter feito mais), bem como pela forma escolhida para o tratamento terminal (Das et al., 2019).

Destaca-se também achados referentes a um maior cuidado com os filhos por parte dos pais de filhos com câncer quando comparados a pais de crianças saudáveis (Long et al., 2013) e essa diferença é notada inclusive dentro do próprio âmbito familiar, onde nota-se uma maior superproteção e receptividade emocional com as crianças doente quando comparadas a irmãos, por exemplo. Isso acarreta uma inversão de que deveria ser algo positivo (o maior cuidado com os filhos), em algo negativo (ciúmes), o que pode desestabilizar o ambiente familiar, e como já exposto acima, prejudicar indiretamente o tratamento.

É indubitável que quando uma criança está sendo tratada de câncer, a vida de toda família muda, notou-se então, que o apoio familiar, foi um dos principais recursos que fizeram com que os pais lidassem melhor com toda a situação. Diante disso, três temas principais surgiram: a) Estar junto é importante, posto que a presença dos pais e irmãos durante o tratamento é fundamental e geram total apoio; b) Encontrar apoio para falar e não falar, de forma que a família notou a necessidade de falar sobre a doença, isso serve como esvaziamento e gera alívio; c) trabalho em equipe, uma vez que todos os membros da família repensaram suas contribuições para a vida familiar e notaram que essa atitude melhorava o cenário por qual estavam passando (Schoors et al., 2019).

Importante citar que os laços sociais próximos como o envolvimento de vizinhos e amigos quando um membro da família está doente, desempenha um papel fundamental no enfrentamento do câncer pediátrico infantil. Além disso, fortes crenças religiosas foram identificadas como fator facilitador no enfrentamento das condições da criança. Independente da religião, a esperança e a fé em um Deus Misericordioso, e este relacionamento com Deus deu forças aos pais para lidar com o diagnóstico (Doumit \& Khoury, 2017).

Ressalta-se que todas as emoções negativas citadas potencializam a tensão criada na relação entre os pais, que apesar de não ser citada por todos os autores como uma emoção negativa, mas praticamente todos eles consideram como algo que interfere tanto no tratamento, como no bem estar parental, e da criança. Associado a isso, considera-se inclusive a necessidade de uma atenção da equipe médica voltada para os pais e para relação entre eles durante o tratamento oncológico, muito embora se reconheça que ainda há uma dificuldade dos médicos em ter atitude e conseguir uma abertura para tratar sobre esse aspecto (Hoogue et al., 2018).

Torna-se cada vez mais importante e necessário esse manejo das relações entre os pais no tratamento, isso porque estudos já confirmaram que casais com menos arestas entre si são mais propensos a ter um filho em tratamento ativo (Rensen et al., 2019a), no entanto, ainda existe uma dificuldade por parte da equipe médica em equilibrar essa necessidade de atenção aos pais com a necessidade de atenção à criança, que apesar de todo o exposto, ainda deve ser prioridade.

Por fim, algumas limitações do presente estudo foram encontradas, como os achados em apenas duas plataformas de dados, apesar da pesquisa ter sido realizada em três, indicativo de escassez de artigos sobre a temática, especialmente em revistas médicas e a necessidade de outros estudos para aprofundar a discussão. 
No mais, as emoções positivas, apesar de igual número, não foram tão exploradas pelos artigos encontrados se comparadas às negativas. Ademais, outra limitação referiu-se à falta de artigos brasileiros, os quais poderiam refletir melhor a realidade do país.

\section{Considerações Finais}

Em suma, o diagnóstico do câncer infantil é um momento muito delicado para toda a família, especialmente para o cuidador principal, geralmente a mãe, pois é ela quem acompanha o filho ou filha ao longo do tratamento. Essa nova realidade exige muito dela e de toda a família e faz aflorar muitos sentimentos negativos e positivos no grupo familiar.

Essas emoções são responsáveis por modificações profundas e irreparáveis em todos os componentes do grupo familiar: para o filho diagnosticado, essa exposição a um evento traumático como o câncer na fase inicial da vida humana guarda relação com o desenvolvimento de distúrbios mórbidos de várias naturezas na idade adulta.

Já para os demais integrantes, seja na relação dos pais com o filho doente, com os demais filhos ou entre eles, notouse uma mudança na dinâmica familiar, com geração de atritos e distanciamento entre eles, figurando, dessa forma, como uma extensão dos danos decorrentes da doença e do seu tratamento.

Torna-se assim muito bem destacado o papel de um acompanhamento psicológico para a criança e demais membros da família no sentido de minimizar os efeitos deletérios causados pelos sentimentos ruins e ampliar o alcance dos bons sentimentos.

Este trabalho não teve a pretensão de esgotar o assunto, mas sim contribuir para uma reflexão acerca dos aspectos emocionais da criança com câncer e de seus familiares.

\section{Referências}

Alves, K. M. C., Comassetto, I., Almeida, T. G., Trezza, M. C. S. F., Silva, J. M. O. \& Magalhães, A. P. N. (2016). The experience of parents of children with cancer in treatment failure conditions. Texto \& Contexto - Enfermagem, 25(2), e2120014. http://dx.doi.org/10.1590/0104-07072016002120014.

Buchbinder, D. K., Fortier, M. A., Osann, K., Wilford, J., Shen, V., Torno, L., Sender, L. S. et al. (2017). Quality of Life Among Parents of Adolescent and Young Adult Brain Tumor Survivors. Journal Of Pediatric Hematology/Oncology, 39(8), 579-584. http://dx.doi.org/10.1097/mph.0000000000000947

Castro, E. H. B. (2010). Revista Mal-estar e Subjetividade, X(3), 971-994. Fortaleza.

Cox, T. (2016). Caregivers reflecting on the early days of childhood cancer. European Journal Of Cancer Care, $27(1)$, e12499. http://dx.doi.org/10.1111/ecc.12499.

D’urso, A., Mastroyannopoulou, K. \& Kirby, A. (2016). Experiences of posttraumatic growth in siblings of children with cancer. Clinical Child Psychology And Psychiatry, 22(2), 301-317. http://dx.doi.org/10.1177/1359104516660749.

Das, K., Khanna, T., Arora, A. \& Agrawal, N. (2019). Parents' acceptance and regret about end of life care for children who died due to malignancy. Supportive Care In Cancer, 28(1), 303-308. http://dx.doi.org/10.1007/s00520-019-04806-0.

Dib, R. V., Gomes, A. M. T., Ramos, R. S., França, L. C. M. \& MARQUES, S. C. (2020). Cancer and its social representations for cancer patients. Research, Society and Development, 9(9), e187997134. 10.33448/rsd-v9i9.7134.

Doumit, M. A. A., Khoury, M. N. (2017). Facilitating and hindering factors for coping with the experience of having a child with cancer: a lebanese perspective. Journal Of Psychosocial Oncology, 35(3), 346-361. http://dx.doi.org/10.1080/07347332.2017.1283654.

Feliciano, S. V. M., Santos, M. O. \& Pombo-de-Oliveira, M. S. (2018). Incidência e Mortalidade por Câncer entre Crianças e Adolescentes: uma revisão narrativa. Revista Brasileira de Cancerologia, 64(3), 389-396. http://dx.doi.org/10.32635/2176-9745.rbc.2018v64n3.45.

Fortier, M. A., Wahi, A., Bruce, C., Maurer, E. L. \& Stevenson, R. (2013). Pain management at home in children with cancer: a daily diary study. Pediatric Blood \& Cancer, 61(6), 1029-1033. http://dx.doi.org/10.1002/pbc.24907.

Granek, L., Rosenberg-Yunger, Z. R. S., Dix, D., Klaassen, R. J., Sung, L., Cairney, J., Klassen, A. F. (2012). Caregiving, single parents and cumulative stresses when caring for a child with cancer. Child: Care, Health and Development, 40(2), 184-194. http://dx.doi.org/10.1111/cch.12008.

Heidegger, M. A. (2002). Ser e tempo (M. S. Cavalcante Trad., 1(9)). Petrópolis, RJ: Vozes.

Hildenbrand, A. K., Clawson, K. J., Alderfer, M. A. \& Marsac, M. L. (2011). Coping With Pediatric Cancer. Journal Of Pediatric Oncology Nursing, 28(6), 344-354. http://dx.doi.org/10.1177/1043454211430823. 
Hooghe, A., Rosenblatt, P., Jongh, S., Bakker, E., Nijkamp, M. \& Rober, P. (2018). "The child is our focus": on couple issues in child oncology treatment. Psycho-Oncology, 27(10), 2482-2487. http://dx.doi.org/10.1002/pon.4855.

Hooghe, A., Rosenblatt., P. C., Vercruysse, T., Cosyns, V., Lambrecht, K., \& Rober, P. (2020). "It's Hard to Talk When Your Child Has a Life Threatening Illness": a qualitative study of couples whose child is diagnosed with cancer. Journal Of Pediatric Oncology Nursing, 37(6), 398-407. http://dx.doi.org/10.1177/1043454220944125.

Instituto Nacional do Câncer (2016). Incidência, mortalidade e morbidade hospitalar por câncer em crianças, adolescentes e adultos jovens no Brasil: informações dos registros de câncer e do sistema de mortalidade. Rio de Janeiro.

Junior, D. C. (2016). The formation of citizens: the pediatrician's role. Jornal de Pediatria, 92(3), 23-29. http://dx.doi.org/10.1016/j.jped.2015.12.002. Kim, M. Y. (2017). Factors Influencing Posttraumatic Growth in Mothers of Children With Cancer. Journal Of Pediatric Oncology Nursing, 34(4), 250-260. http://dx.doi.org/10.1177/1043454217697021.

Kostak, M., Semerci, R., Eren, T., Avci, G. \& Savran, F. (2019). Life Experiences of Adolescents with Cancer in Turkey: a phenomenological study. Journal Of The Pakistan Medical Association, 69(10), 1464-1469. 10.5455/JPMA.300670

Lazar, M. B., Musek, J. (2020). Well-being in parents of children with cancer: the impact of parental personality, coping, and the child's quality of life. Scandinavian Journal Of Psychology, 61(5), 652-662. http://dx.doi.org/10.1111/sjop.12653.

Long, K. A., Keeley, L., Reiter-purtill, J., Vannatta, K., Gerhardt, C. A. \& Noll, R. B. (2013). Child-rearing in the context of childhood cancer: perspectives of parents and professionals. Pediatric Blood \& Cancer, 61(2), 326-332. http://dx.doi.org/10.1002/pbc.24556.

Molinaro, M. L., Fletcher, P. C. (2018). The Balancing Act: mothers experiences of providing care to their children with cancer. Journal Of Pediatric Oncology Nursing, 35(6), 439-446. http://dx.doi.org/10.1177/1043454218794667.

Moreira-Dias, P. L., Silva, I. P. (2018). A Utilização do Brinquedo durante o Tratamento de Crianças com Câncer: percepções da equipe multidisciplinar. Revista Brasileira de Cancerologia, 64(3), 311-318. http://dx.doi.org/10.32635/2176-9745.rbc.2018v64n3.28.

Nolbris, M. J., Enskär, K. \& Hellström, A. (2014). Grief Related to the Experience of Being the Sibling of a Child With Cancer. Cancer Nursing, 37(5), E1E7. http://dx.doi.org/10.1097/ncc.0b013e3182a3e585.

Peterson, R. K., Chung, J. \& Barrera, M. (2019). Emotional symptoms and family functioning in caregivers of children with newly diagnosed leukemia/lymphomas and solid tumors: short : term changes and related demographic factors. Pediatric Blood \& Cancer, 67(2). http://dx.doi.org/10.1002/pbc.28059.

Popp, J. M., Conway, M. \& Pantaleao, A. (2015). Parents' Experience With Their Child's Cancer Diagnosis. Journal Of Pediatric Oncology Nursing, 32(4), 253-260. http://dx.doi.org/10.1177/1043454214563404.

Quintana, A. M., Wottrich, S. H., Camargo, V. P., Cherer, E. Q. \& Ries, P. K. (2011). Lutos e lutas: reestruturações familiares diante do câncer em uma criança/adolescente. Psicologia Argumento, 29(65), 143-154. http://dx.doi.org/10.7213/psicolargum.v29i65.20035.

Rensen, N., Steur, L. M. H., Schepers, S. A., Merks, J. H. M., Moll, A. C., Kaspers, G. J. L., Van litsenburg, R. R. L. et al. (2019a). Determinants of healthrelated quality of life proxy rating disagreement between caregivers of children with cancer. Quality Of Life Research, $29(4), 901-912$. http://dx.doi.org/10.1007/s11136-019-02365-9.

Rensen, N., Steur, L. M. H., Schepers, S. A., Merks, J. H. M., Moll, A. C., Kaspers, G. J. L., Van litsenburg, R. R. L. et al. (2019b). Gender-specific differences in parental health-related quality of life in childhood cancer. Pediatric Blood \& Cancer, 66(7), e27728. http://dx.doi.org/10.1002/pbc.27728.

Santos, M. R., Wiegand, D. L., Sá, N. N., Misko, M. D. \& Szylit, R. (2019). From hospitalization to grief: meanings parents assign to their relationships with pediatric oncology professional. Rev Esc Enferm USP. 53, e03521. http://dx.doi.org/10.1590/S1980-220X2018049603521.

Shi, L., Gao, Y., Zhao, J., Cai, R., Zhang, P., Hu, Y., Li, Z. et al. (2016). Prevalence and predictors of posttraumatic stress symptoms in parents of children with ongoing treatment for cancer in South China: a multi-centered cross-sectional study. Supportive Care In Cancer, 25(4), 1159-1167. http://dx.doi.org/10.1007/s00520-016-3506-6.

Sisk, B. A., Kang, T. I. \& Mack, J. W. (2019). The evolution of regret: decision-making for parents of children with cancer. Supportive Care In Cancer, 28(3), 1215-1222. http://dx.doi.org/10.1007/s00520-019-04933-8

Sousa, M. N. A. (2016). Revisão Integrativa da Literatura: esclarecendo o método. In: Sousa, M. N. A.; Santos, E. V. L. Medicina e pesquisa: um elo possível. (pp. 345-358). Curitiba: Editora CRV.

Van Schoors, M., Mol, J., Verhofstadt, L. L., Goubert, L. \& Van parys, H. (2020). The family practice of support-giving after a pediatric cancer diagnosis: a multi-family member interview analysis. European Journal Of Oncology Nursing, 44, 101712. http://dx.doi.org/10.1016/j.ejon.2019.101712.

Watanabe, A., Nunes, T. \& Abreu, G. (2013). Japanese parents' perception of disclosing the diagnosis of cancer to their children. Clinical Child Psychology And Psychiatry, 19(1), 125-138. http://dx.doi.org/10.1177/1359104512470599. 